\title{
FUNDAMENTAL PERFORMANCE LIMITATIONS IN ESTIMATION PROBLEMS*
}

\author{
LI QIU ${ }^{\dagger}$ ZHIYUAN RENं, AND JIE CHEN ${ }^{\S}$
}

\begin{abstract}
In this paper, we address the performance limitation issues in estimation problems. Our purpose is to explicitly relate the best achievable estimation errors with simple plant characteristics. In particular, we study performance limitations in achieving four objectives: (1) estimating the output of an LTI system from its version corrupted by a white noise, (2) estimating a Brownian motion from its version distorted by an LTI system, (3) estimating the output of an LTI system from its version corrupted by a Brownian noise, (4) estimating a white noise from its version distorted by an LTI system.
\end{abstract}

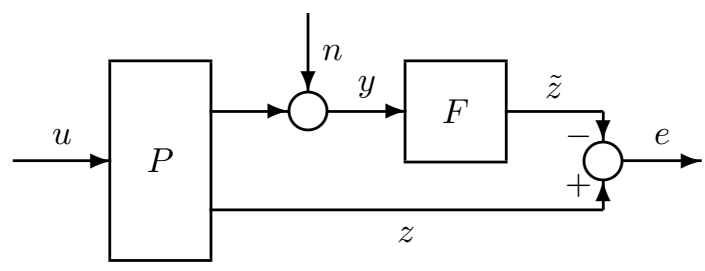

FIG. 1. A general estimation problem

1. Introduction. A standard estimation problem can often be schematically shown by Fig. 1 . Here $P=\left[\begin{array}{c}G \\ H\end{array}\right]$ is an LTI plant, $u$ is the input to the plant, $n$ is the measurement noise, $z$ is the signal to be estimated, $y$ is the measured signal, $\tilde{z}$ is the estimate of $z$. Often $u$ and $n$ are modelled as stochastic processes with known means and covariances. We can assume, without loss of generality, that the means of the stochastic processes are zero. The objective is to design LTI filter $F$ so that the steady state error variance

$$
V=\lim _{t \rightarrow \infty} \boldsymbol{E}\left[e(t)^{\prime} e(t)\right]
$$

is small. Clearly, for $V$ to be finite for nontrivial $u$ and $n$, it is necessary that $F \in \mathcal{R} \mathcal{H}_{\infty}$ and $H-F G \in \mathcal{R H}_{\infty}$. This condition is also necessary and sufficient for the error to be bounded for arbitrary initial conditions of $P$ and $F$, i.e., for the filter to be a

\footnotetext{
${ }^{*}$ Received on February 18, 2002; accepted for publication on September 10, 2002. This work is supported by the Research Grants Council of Hong Kong under grant HKUST6131/98E and National Science Foundation of USA under grant ECS-9912533.

${ }^{\dagger}$ Department of Electrical and Electronic Engineering, Hong Kong University of Science and Technology, Clear Water Bay, Kowloon, Hong Kong. E-mail: eeqiu@ee.ust.hk

${ }_{\ddagger}^{\ddagger}$ Department of Electrical \& Computer Engineering, Carnegie Mellon University, Pittsburgh, PA 15213-3890 USA. E-mail: zren@andrew.ucr.edu

$\S$ Department of Electrical Engineering, College of Engineering, University of California,Riverside, CA 92521-0425 USA. E-mail: jchen@ee.ucr.edu
} 
bounded error estimator (BEE). There is an extensive theory on the optimal design of the filter $F$ to minimize $V$, see for example $[1,2,6]$. The optimal error variance is then given by

$$
V^{*}=\inf _{F, H-F G \in \mathcal{R} \mathcal{H}_{\infty}} V .
$$

Our interest in this paper is not on how to find the optimal filter $F$, which is addressed by the standard optimal filtering theory. Rather, we are interested in relating $V^{*}$ with some simple characteristics of the plant $P$ in some important special cases. Since $V^{*}$ gives a fundamental limitation in achieving certain performance objectives in filtering problems, the simple relationship between $V^{*}$ and the plant characteristics, in addition to providing deep understanding and insightful knowledge on estimation problems, can be used to access the quality of different designs and to ascertain impossible design objectives before a design is carried out.

The variance $V$ gives an overall measure on the size of the steady state estimation error. Sometimes, we may wish to focus on some detailed features of the error. For example, we may wish to see the steady state variance of the $i$-th entry of the error, which is given by

$$
V_{i}=\lim _{t \rightarrow \infty} \boldsymbol{E}\left[e_{i}(t)^{2}\right]
$$

More generally, we may wish to investigate the variance of the projection of the estimation error on certain direction. This variance then gives a measure of the error in a particular direction. Assume that $z(t), \tilde{z}(t), e(t) \in \mathbb{R}^{m}$. Let $\xi \in \mathbb{R}^{m}$ be a vector of unit length representing a direction in $\mathbb{R}^{m}$. Then the projection of $e(t)$ to the direction represented by $\xi$ is given by $\xi^{\prime} e(t)$ and its steady state variance is given by

$$
V_{\xi}=\lim _{t \rightarrow \infty} \boldsymbol{E}\left[\left(\xi^{\prime} e(t)\right)^{2}\right] .
$$

The best achievable error in $\xi$ direction is then given by

$$
V_{\xi}^{*}=\inf _{F, H-F G \in \mathcal{R} \mathcal{H}_{\infty}} V_{\xi}
$$

The optimal or near optimal filter in minimizing $V_{\xi}$ in general depends on $\xi$. This very fact may limit the usefulness of $V_{\xi}^{*}$, since we are usually more interested in the directional error information under an optimal or near optimal filter designed for all directions, i.e., designed to minimize $V$. Let $\left\{F_{k}\right\}$ be a sequence of filters satisfying $F_{k}, H-F_{k} G \in \mathcal{R H}_{\infty}$ such that the corresponding sequence of errors $\left\{e_{k}\right\}$ satisfies

$$
V=\lim _{k \rightarrow \infty} \lim _{t \rightarrow \infty} \boldsymbol{E}\left[e_{k}(t) e_{k}(t)^{\prime}\right] .
$$

Then we are more interested in

$$
V^{*}(\xi)=\lim _{k \rightarrow \infty} \boldsymbol{E}\left[\left(\xi^{\prime} e_{k}(t)\right)^{2}\right] .
$$


In this paper, we will also give the relationship between $V_{\xi}^{*}, V^{*}(\xi)$ and simple characteristics of the plant $P$ for the same cases when that for $V^{*}$ is considered.

The performance limitations in estimation have been studied recently in $[4,5$, $11,12]$ in various settings. In $[4,5,11]$, sensitivity and complimentary sensitivity functions of an estimation problem are defined and it is shown that they have to satisfy certain integral constraints independent of filter design. In [12], a time domain technique is used to study the performance limitations in some special cases when one of $n$ and $u$ is diminishingly small and the other one is either a white noise or a Brownian motion.

This paper addresses similar problems as in [12] and their extensions, but studies them from a pure input output point of view using frequency domain techniques. We also study them in more detail by providing directional information on the best errors. The results obtained are dual to those in $[3,7]$ where the performance limitations of tracking and regulation problems are considered. The new investigation provides more insights into the performance limitations of estimation problems.

This paper is organized as follows: Section 2 provides background materials on transfer matrix factorizations which exhibit directional properties of each nonminimum phase zero and antistable pole. Section 3 relates the performance limitation in estimating a signal from its corrupted version by a white noise to the antistable modes, as well as their directional properties, of the signal. Section 4 relates the performance limitation in estimating a Brownian motion from its version distorted by an LTI system to the nonminimum phase zeros of the system, as well as their directional properties. Section 5 addresses a similar problem as Section 3 except that the noise is assumed to be a Brownian motion. Section 6 addresses a similar problem as section 4 except that the process to be estimated is assumed to be a white noise. Section 7 gives concluding remarks.

2. Preliminaries. Let $G$ be a continuous time FDLTI system. We will use the same notation $G$ to denote its transfer matrix. Assume that $G$ is left invertible. The poles and zeros of $G$, including multiplicity, are defined according to its SmithMcMillan form. A zero of $G$ is said to be nonminimum phase if it has positive real part. $G$ is said to be minimum phase if it has no nonminimum phase zero; otherwise, it is said to be nonminimum phase. A pole of $G$ is said to be antistable if it has a positive real part. $G$ is said to be semistable if it has no antistable pole; otherwise strictly unstable. A stable $G$ is said to be unitary if $G(j \omega) G(j \omega)^{*}=G(j \omega)^{*} G(j \omega)=I$.

Suppose that $G$ is stable and $z$ is a nonminimum phase zero of $G$. Then, there exists a vector $u$ of unit length such that

$$
G(z) u=0 .
$$

We call $u$ a (right or input) zero vector corresponding to the zero $z$. Let the nonminimum phase zeros of $G$ be ordered as $z_{1}, z_{2}, \ldots, z_{\nu}$. Let also $\eta_{1}$ be a zero vector 
corresponding to $z_{1}$. Define

$$
G_{1}(s)=I-\frac{2 \operatorname{Re} z_{1}}{s+z_{1}^{*}} \eta_{1} \eta_{1}^{*}
$$

Note that $G_{1}$ is so constructed that it is unitary, has only one zero at $z_{1}$ with $\eta_{1}$ as a zero vector. Now $G G_{1}^{-1}$ has zeros $z_{2}, z_{3}, \ldots, z_{\nu}$. Find a zero vector $\eta_{2}$ corresponding to the zero $z_{2}$ of $G G_{1}^{-1}$, and define

$$
G_{2}(s)=I-\frac{2 \operatorname{Re} z_{2}}{s+z_{2}^{*}} \eta_{2} \eta_{2}^{*}
$$

It follows that $G G_{1}^{-1} G_{2}^{-1}$ has zeros $z_{3}, z_{4}, \ldots, z_{\nu}$. Continue this process until $\eta_{1}, \ldots, \eta_{\nu}$ and $G_{1}, \ldots, G_{\nu}$ are obtained. Then we have one vector corresponding to each nonminimum phase zero, and the procedure yields a factorization of $G$ in the form of

$$
G=G_{0} G_{\nu} \cdots G_{1}
$$

where $G_{0}$ has no nonminimum phase zeros and

$$
G_{i}(s)=I-\frac{2 \operatorname{Re} z_{i}}{s+z_{i}^{*}} \eta_{i} \eta_{i}^{*}
$$

Since $G_{i}$ is unitary, has the only zero at $z_{i}$, and has $\eta_{i}$ as a zero vector corresponding to $z_{i}$, it can be considered as a generalization of the standard scalar Blaschke factor, see [10] for example, and hence will be called a matrix Blaschke factor. Accordingly, the product

$$
G_{z}=G_{\nu} \cdots G_{1}
$$

will be called a matrix Blaschke product. The vectors $\eta_{1}, \ldots, \eta_{\nu}$ will be called zero Blaschke vectors of $G$ corresponding to the nonminimum phase zeros $z_{1}, z_{2}, \ldots, z_{\nu}$. Keep in mind that these vectors depend on the order of the nonminimum phase zeros. One might be concerned with the possible complex coefficients appearing in $G_{i}$ when some of the nonminimum phase zeros are complex. However, if we order a pair of complex conjugate nonminimum phase zeros adjacently, then the corresponding pair of Blaschke factors will have complex conjugate coefficient and their product is then real rational and this also leads to real rational $G_{0}$.

The choice of $G_{i}$ as in (2) seems ad hoc notwithstanding that $G_{i}$ has to be unitary, have the only zero at $z_{i}$ and have $\eta_{i}$ as a zero vector corresponding to $z_{i}$. Another choice, among infinite many possible ones, is

$$
G_{i}(s)=I-\frac{2 \operatorname{Re} z_{i}}{z_{i}} \frac{s}{s+z_{i}^{*}} \eta_{i} \eta_{i}^{*},
$$

and if this choice is adopted, the same procedure can be used to find a factorization of the form (1). Of course, in this case the Blaschke vectors are not the same. We 
see that for the first choice $G_{i}(\infty)=I$, whereas for the second choice $G_{i}(0)=I$. We will use both choices in the following. For this purpose, we will call the factorization resulting from the first choice (2) of Type I and that from the second choice (3) of type II.

For an unstable $G$, there exist stable real rational matrix functions

$$
\left[\begin{array}{cc}
\tilde{X} & -\tilde{Y} \\
-\tilde{N} & \tilde{M}
\end{array}\right],\left[\begin{array}{cc}
M & Y \\
N & X
\end{array}\right]
$$

such that

$$
G=N M^{-1}=\tilde{M}^{-1} \tilde{N}
$$

and

$$
\left[\begin{array}{cc}
\tilde{X} & -\tilde{Y} \\
-\tilde{N} & \tilde{M}
\end{array}\right]\left[\begin{array}{cc}
M & Y \\
N & X
\end{array}\right]=I .
$$

This is called a doubly coprime factorization of $G$. Note that the nonminimum phase zeros of $G$ are the nonminimum phase zeros of $\tilde{N}$ and the antistable poles of $G$ are the nonminimum phase zeros of $\tilde{M}$. If we order the antistable poles of $G$ as $p_{1}, p_{2}, \ldots, p_{\mu}$ and the nonminimum phase zeros of $G$ as $z_{1}, z_{2}, \ldots, z_{\nu}$, then $\tilde{M}$ and $\tilde{N}$ can be factorized as

$$
\begin{aligned}
\tilde{M} & =\tilde{M}_{0} \tilde{M}_{\mu} \cdots \tilde{M}_{1} \\
\tilde{N} & =\tilde{N}_{0} \tilde{N}_{\nu} \cdots \tilde{N}_{1}
\end{aligned}
$$

with

$$
\tilde{M}_{i}(s)=I-\frac{2 \operatorname{Re} p_{i}}{s+p_{i}^{*}} \zeta_{i} \zeta_{i}^{*}, \quad i=1,2, \ldots, \mu
$$

or

$$
\tilde{M}_{i}(s)=I-\frac{2 \operatorname{Re} p_{i}}{p_{i}} \frac{s}{s+p_{i}^{*}} \zeta_{i} \zeta_{i}^{*}, \quad i=1,2, \ldots, \mu
$$

and

$$
\tilde{N}_{i}(s)=I-\frac{2 \operatorname{Re} z_{i}}{s+z_{i}^{*}} \eta_{i} \eta_{i}^{*}, \quad i=1,2, \ldots, \nu
$$

or

$$
\tilde{N}_{i}(s)=I-\frac{2 \operatorname{Re} z_{i}}{z_{i}} \frac{s}{s+z_{i}^{*}} \eta_{i} \eta_{i}^{*}
$$

where $\zeta_{1}, \zeta_{2}, \ldots, \zeta_{\mu}$ are zero Blaschke vectors (of type I or II) of $\tilde{M}$ and $\eta_{1}, \eta_{2}, \ldots, \eta_{\nu}$ are those of $\tilde{N}$. Here also $\tilde{N}_{0}$ and $\tilde{M}_{0}$ have no nonminimum phase zeros. 
Consequently, for any real rational matrix $G$ with nonminimum phase zeros $z_{1}, z_{2}, \ldots, z_{\nu}$ and antistable poles $p_{1}, p_{2}, \ldots, p_{\mu}$, it can always be factorized to

$$
G=G_{p}^{-1} G_{0} G_{z}
$$

as shown in Fig. 2, where

$$
\begin{array}{rlll}
G_{p}(s)=\prod_{i=1}^{\mu}\left[I-\frac{2 \operatorname{Re} p_{i}}{s+p_{i}^{*}} \zeta_{i} \zeta_{i}^{*}\right] & \text { or } & \prod_{i=1}^{\mu}\left[I-\frac{2 \operatorname{Re} p_{i}}{p_{i}} \frac{s}{s+p_{i}^{*}} \zeta_{i} \zeta_{i}^{*}\right] \\
G_{z}(s)=\prod_{i=1}^{\nu}\left[I-\frac{2 \operatorname{Re} z_{i}}{s+z_{i}^{*}} \eta_{i} \eta_{i}^{*}\right] & \text { or } & \prod_{i=1}^{\nu}\left[I-\frac{2 \operatorname{Re} z_{i}}{z_{i}} \frac{s}{s+z_{i}^{*}} \eta_{i} \eta_{i}^{*}\right]
\end{array}
$$

and $G_{0}$ is a real rational matrix with neither nonminimum phase zero nor antistable pole. Although coprime factorizations of $G$ are not unique, this nonuniqueness does not affect factorization (8). Here $\eta_{1}, \eta_{2}, \ldots, \eta_{\nu}$ are called zero Blaschke vectors (of type I or II) and $\zeta_{1}, \zeta_{2}, \ldots, \zeta_{\nu}$ pole Blaschke vectors (of type I or II) of $G$.

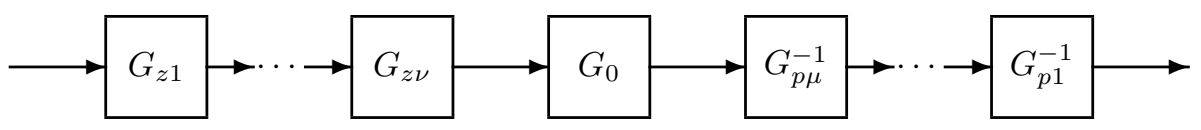

FIG. 2. Cascade factorization

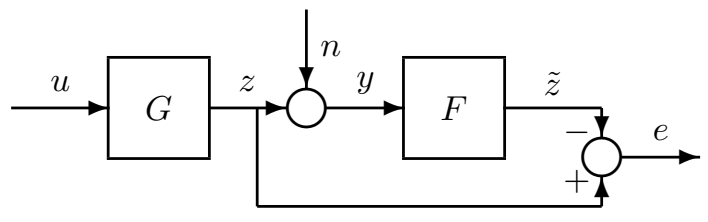

FIG. 3. Estimation under measurement noise

3. Estimation under White Measurement Noise. Consider the estimation problem shown in Fig. 3. Here $G$ is a given FDLTI plant, and $n$ is a standard white noise. Assume that $u$ is a white noise with vanishingly small covariance, i.e., $\boldsymbol{E}\left[u(t) u(t)^{\prime}\right]=\epsilon^{2} \delta(t) I$ and $\epsilon \rightarrow 0$. The purpose is to design a stable LTI filter $F$ such that it generates an estimate $\tilde{z}$ of the true output $z$ using the corrupted output $y$. This problem is clearly a special case of the general estimation problem stated in Sect. 1 with $P=\left[\begin{array}{l}G \\ G\end{array}\right]$. Since $\epsilon$ may be nonzero, we need to have $F, G-F G \in \mathcal{R} \mathcal{H}_{\infty}$. On the other hand, since $\epsilon$ is vanishingly small, the error of estimation can be approximated by $F n$ in a practical sense. Since $n$ is a standard white noise, the steady state variance of the error is then given by

$$
V=\|F\|_{2}^{2}
$$


where $\|\cdot\|_{2}$ is the $\mathcal{H}_{2}$ norm. If we want $V$ to be finite, we need to have $F(\infty)=0$, in addition to $F, G-F G \in \mathcal{R} \mathcal{H}_{\infty}$. Therefore

$$
V^{*}=\inf _{F, G-F G \in \mathcal{R} \mathcal{H}}^{\infty, F(\infty)=0},\|F\|_{2}^{2}
$$

Let $G=\tilde{M}^{-1} \tilde{N}$ be a left coprime factorization of $G$. Then $F \in \mathcal{R H}_{\infty}$ and $G-F G=(I-F) G=(I-F) \tilde{M}^{-1} \tilde{N} \in \mathcal{R} \mathcal{H}_{\infty}$ if and only if $I-F=Q \tilde{M}$ for some $Q \in \mathcal{R} \mathcal{H}_{\infty}$. Therefore

$$
V^{*}=\inf _{Q \in \mathcal{R} \mathcal{H}_{\infty}, Q(\infty) \tilde{M}(\infty)=I}\|I-Q \tilde{M}\|_{2}^{2}
$$

Now assume that $G$ has antistable poles $p_{1}, p_{2}, \ldots, p_{\mu}$ with $\zeta_{1}, \zeta_{2}, \ldots, \zeta_{\mu}$ be the corresponding pole Blaschke vectors of type I. Then $\tilde{M}$ has factorization

$$
\tilde{M}=\tilde{M}_{0} \tilde{M}_{\mu} \cdots \tilde{M}_{1}
$$

where

$$
\tilde{M}_{i}(s)=I-\frac{2 \operatorname{Re} p_{i}}{s+p_{i}^{*}} \zeta_{i} \zeta_{i}^{*}
$$

Since $\tilde{M}_{i}(\infty)=I, i=1,2, \ldots, \mu$, it follows that $Q(\infty) \tilde{M}(\infty)=I$ is equivalent to $Q(\infty) \tilde{M}_{0}(\infty)=I$. Hence, by using the facts that $\tilde{M}_{i}, i=1,2, \ldots, \mu$, are unitary operators in $\mathcal{L}_{2}$ and that $\tilde{M}_{1}^{-1} \cdots \tilde{M}_{\mu}^{-1}-I \in \mathcal{H}_{2}^{\perp}$ and $I-Q \tilde{M}_{0} \in \mathcal{H}_{2}$, we obtain

$$
\begin{aligned}
V^{*} & =\inf _{Q \in \mathcal{R} \mathcal{H}_{\infty}, Q(\infty) \tilde{M}_{0}(\infty)=I}\left\|I-Q \tilde{M}_{0} \tilde{M}_{\mu} \cdots \tilde{M}_{1}\right\|_{2}^{2} \\
& =\inf _{Q \in \mathcal{R} \mathcal{H}_{\infty}, Q(\infty) \tilde{M}_{0}(\infty)=I}\left\|\tilde{M}_{1}^{-1} \cdots \tilde{M}_{\mu}^{-1}-I+I-Q \tilde{M}_{0}\right\|_{2}^{2} \\
& =\left\|\tilde{M}_{1}^{-1} \cdots \tilde{M}_{\mu}^{-1}-I\right\|_{2}^{2}+\inf _{Q \in \mathcal{R} \mathcal{H}_{\infty}, Q(\infty) \tilde{M}_{0}(\infty)=I}\left\|I-Q \tilde{M}_{0}\right\|_{2}^{2} .
\end{aligned}
$$

Since $\tilde{M}_{0}$ is minimum phase with invertible $\tilde{M}_{0}(\infty)$, there exists a sequence $\left\{Q_{k}\right\} \in$ $\mathcal{R} \mathcal{H}_{\infty}$ with $Q_{k}(\infty) \tilde{M}_{0}(\infty)=I$ such that $\lim _{k \rightarrow \infty}\left\|I-Q_{k} \tilde{M}_{0}\right\|_{2}=0$. This shows

$$
\begin{aligned}
V^{*} & =\left\|\tilde{M}_{1}^{-1} \cdots \tilde{M}_{\mu}^{-1}-I\right\|_{2}^{2} \\
& =\left\|\tilde{M}_{2}^{-1} \cdots \tilde{M}_{\mu}^{-1}-I+I-\tilde{M}_{1}\right\|_{2}^{2} \\
& =\left\|\tilde{M}_{2}^{-1} \cdots \tilde{M}_{\mu}^{-1}-I\right\|_{2}^{2}+\left\|I-\tilde{M}_{1}\right\|_{2}^{2} \\
& =\sum_{i=1}^{\mu}\left\|I-\tilde{M}_{i}\right\|_{2}^{2} \\
& =2 \sum_{i=1}^{\mu} p_{i} .
\end{aligned}
$$

Here the first equality follows from that $\tilde{M}_{1}$ is a unitary operator in $\mathcal{L}_{2}$, the second from that $\tilde{M}_{2}^{-1} \cdots \tilde{M}_{\mu}^{-1}-I \in \mathcal{H}_{2}^{\perp}$ and $I-\tilde{M}_{1} \in \mathcal{H}_{2}$, the third from repeating the 
underlying procedure in the first and second equalities, and the last from straightforward computation. The above derivation shows that an arbitrarily near optimal $Q$ can be chosen from the sequence $\left\{Q_{k}\right\}$. Therefore

$$
V^{*}(\xi)=\lim _{k \rightarrow \infty}\left\|\xi^{\prime}\left(I-Q_{k} \tilde{M}_{0} \tilde{M}_{\mu} \cdots \tilde{M}_{1}\right)\right\|_{2}^{2}
$$

The same reasoning as in the above derivation gives

$$
V^{*}(\xi)=\sum_{i=1}^{\mu}\left\|\xi\left(I-\tilde{M}_{i}\right)\right\|_{2}^{2}=2 \sum_{i=1}^{\mu} p_{i} \cos ^{2} \angle\left(\xi, \zeta_{i}\right) .
$$

The last equality follows from straightforward computation.

The directional steady state error variance with an arbitrary $F$ is

$$
V_{\xi}=\left\|\xi^{\prime} F\right\|_{2}^{2}
$$

and the optimal directional steady state error variance is

$$
\begin{aligned}
V_{\xi}^{*} & =\inf _{F, G-F G \in \mathcal{R} \mathcal{H}_{\infty}}\left\|\xi^{\prime} F\right\|_{2}^{2} \\
& =\inf _{Q \in \mathcal{R} \mathcal{H}_{\infty}, Q(\infty) \tilde{M}_{0}(\infty)=I}\left\|\xi^{\prime}\left(I-Q \tilde{M}_{0} \tilde{M}_{\mu} \cdots \tilde{M}_{1}\right)\right\|_{2}^{2} .
\end{aligned}
$$

By following an almost identical derivation as the non-directional case, we can show that the same sequence $\left\{Q_{k}\right\}$ giving near optimal solutions there also gives near optimal solutions here for every $\xi \in \mathbb{R}^{m}$. Hence,

$$
V_{\xi}^{*}=V^{*}(\xi)=2 \sum_{i=1}^{\mu} p_{i} \cos ^{2} \angle\left(\xi, \zeta_{i}\right) .
$$

We have thus established the following theorem.

Theorem 1. Let $G$ 's antistable poles be $p_{1}, p_{2}, \ldots, p_{\mu}$ with $\zeta_{1}, \zeta_{2}, \ldots, \zeta_{\mu}$ being the corresponding pole Blaschke vectors of type I. Then

$$
V^{*}=2 \sum_{i=1}^{\mu} p_{i}
$$

and

$$
V_{\xi}^{*}=V^{*}(\xi)=2 \sum_{i=1}^{\mu} p_{i} \cos ^{2} \angle\left(\xi, \zeta_{i}\right) .
$$

This theorem says that to estimate a signal from its version corrupted by a standard while noise, the best achievable steady state error variance is proportional to the sum of the antistable modes of the signal to be estimated. The best achievable directional steady state error variance depends, in addition, on the directional characteristics of the antistable modes. 


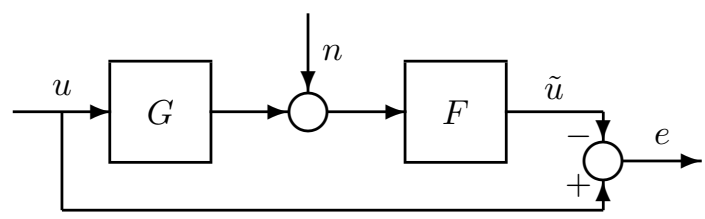

FIG. 4. Estimation of a stochastic process

4. Estimation of Brownian Motion. Consider the estimation problem shown in Fig. 4. Here $G$ is a given FDLTI plant, $u$ is the input to the plant which is assumed to be a Brownian motion process, i.e., the integral of a standard white noise, which can be used to model a slowly varying "constant", $n$ is a white noise with vanishingly small covariance, i.e., $\boldsymbol{E}\left[n(t) n(t)^{\prime}\right]=\epsilon^{2} \delta(t) I$ and $\epsilon \rightarrow 0$. Assume that $G(0)$ is left invertible. The objective is to design an LTI filter $F$ such that it measures the output of $G$ and generates an estimate $\tilde{u}$ of $u$. This problem is clearly a special case of the general estimation problem stated in Sect. 1 with $P=\left[\begin{array}{c}G \\ I\end{array}\right]$. Since $\epsilon$ is nonzero, we need constraints $F, I-F G \in \mathcal{R} \mathcal{H}_{\infty}$. On the other hand, since $\epsilon$ is vanishingly small, the error of estimation can be approximated by $(I-F G) u$ in a practical sense. Since $u$ is a Brownian process, the variance of the error is given by

$$
V=\|(I-F G) U\|_{2}
$$

where $U(s)=\frac{1}{s} I$ is the transfer matrix of $m$ channels of integrators. If we want $V$ to be finite, we need to have $I-F(0) G(0)=0$, in addition to $F, I-F G \in \mathcal{R} \mathcal{H}_{\infty}$. This requires $G(0)$, the DC gain of of $G$, to be left invertible, which will be assumed. Equivalently, we need to have $F, F G \in \mathcal{H}_{\infty}$ and $F(0) G(0)=I$. Therefore,

$$
V^{*}=\inf _{F, F G \in \mathcal{R} \mathcal{H}_{\infty}, F(0) G(0)=I}\|(I-F G) U\|_{2}^{2} .
$$

Let $G=\tilde{M}^{-1} \tilde{N}$ be a left coprime factorization of $G$. Then it is easy to see that $F, F G \in \mathcal{H}_{\infty}$ is equivalent to $F=Q \tilde{M}$ for some $Q \in \mathcal{H}_{\infty}$. Hence

$$
V^{*}=\inf _{Q \in \mathcal{R H}_{\infty}, Q(0) \tilde{N}(0)=I}\|(I-Q \tilde{N}) U\|_{2}^{2} .
$$

Now let $G$ have nonminimum phase zeros $z_{1}, z_{2}, \ldots, z_{\nu}$ with $\eta_{1}, \eta_{2}, \ldots, \eta_{\nu}$ being the corresponding input Blaschke vectors of type II. Then $\tilde{N}$ has factorizations

$$
\tilde{N}=\tilde{N}_{0} \tilde{N}_{\nu} \cdots \tilde{N}_{1}
$$

where

$$
\tilde{N}_{i}=I-\frac{2 \operatorname{Re} z_{i}}{z_{i}} \frac{s}{s+z_{i}^{*}} \eta_{i} \eta_{i}^{*}
$$


Since $\tilde{N}_{i}(0)=I, i=1,2, \ldots, \nu$, it follows that $Q(0) \tilde{N}(0)=I$ is equivalent to $Q(0) \tilde{N}_{0}(0)=I$. Hence, by using the facts that $\tilde{N}_{i}, i=1,2, \ldots, \nu$, are unitary operators in $\mathcal{L}_{2}$ and that $\tilde{N}_{1}^{-1} \cdots \tilde{N}_{\nu}^{-1}-I \in \mathcal{H}_{2}^{\perp}$ and $I-Q \tilde{N}_{0} \in \mathcal{H}_{2}$, we obtain

$$
\begin{aligned}
V^{*} & =\inf _{Q \in \mathcal{R} \mathcal{H}_{\infty}, Q(0) \tilde{N}_{0}(0)=I}\left\|\left(I-Q \tilde{N}_{0} \tilde{N}_{\nu} \cdots \tilde{N}_{1}\right) U\right\|_{2}^{2} \\
& =\inf _{Q \in \mathcal{R} \mathcal{H}_{\infty}, Q(0) \tilde{N}_{0}(0)=I}\left\|\left(\tilde{N}_{1}^{-1} \tilde{N}_{2}^{-1} \cdots \tilde{N}_{\nu}-I\right) U+\left(I-Q \tilde{N}_{0}\right) U\right\|_{2}^{2} \\
& =\left\|\left(\tilde{N}_{1}^{-1} \tilde{N}_{2}^{-1} \cdots \tilde{N}_{\nu}-I\right) U\right\|_{2}^{2}+\inf _{Q \in \mathcal{R} \mathcal{H}_{\infty}, Q(0) \tilde{N}_{0}(0)=I}\left\|\left(I-Q \tilde{N}_{0}\right) U\right\|_{2}^{2} .
\end{aligned}
$$

Since $\tilde{N}_{0}$ is minimum phase with invertible $\tilde{N}(0)$, there exists a sequence $\left\{Q_{k}\right\} \in \mathcal{R} \mathcal{H}_{\infty}$ with $Q_{k}(0) \tilde{N}_{0}(0)=I$ such that $\lim _{k \rightarrow \infty}\left\|\left(I-Q_{k} \tilde{N}_{0}\right) U\right\|=0$. This shows

$$
\begin{aligned}
V^{*} & =\left\|\left(\tilde{N}_{1}^{-1} \cdots \tilde{N}_{\nu}^{-1}-I\right) U\right\|_{2}^{2} \\
& =\left\|\left(\tilde{N}_{2}^{-1} \cdots \tilde{N}_{\nu}^{-1}-I+I-\tilde{N}_{1}\right) U\right\|_{2}^{2} \\
& =\left\|\left(\tilde{N}_{2}^{-1} \cdots \tilde{N}_{\nu}^{-1}-I\right) U\right\|_{2}^{2}+\left\|\left(I-\tilde{M}_{1}\right) U\right\|_{2}^{2} \\
& =\sum_{i=1}^{\nu}\left\|\left(I-\tilde{N}_{i}\right) U\right\|_{2}^{2} \\
& =2 \sum_{i=1}^{\nu} \frac{1}{z_{i}} .
\end{aligned}
$$

Here the first equality follows from that $\tilde{N}_{1}$ is a unitary operator in $\mathcal{L}_{2}$, the second from that $\left(\tilde{N}_{2}^{-1} \cdots \tilde{N}_{\nu}^{-1}-I\right) U \in \mathcal{H}_{2}^{\perp}$ and $\left(I-\tilde{N}_{1}\right) U \in \mathcal{H}_{2}$, the third from repeating the underlying procedure in the first and second equalities, and the last from straightforward computation.

The above derivation shows that an arbitrarily near optimal $Q$ can be chosen from the sequence $\left\{Q_{k}\right\}$. Therefore

$$
V^{*}(\xi)=\lim _{k \rightarrow \infty}\left\|\xi^{\prime}\left(I-Q_{k} \tilde{N}_{0} \tilde{N}_{\nu} \cdots \tilde{N}_{1}\right) U\right\|_{2}^{2} .
$$

The same reasoning as in the above derivation gives

$$
V^{*}(\xi)=\sum_{i=1}^{\mu}\left\|\xi\left(I-\tilde{N}_{i}\right) U\right\|_{2}^{2}=2 \sum_{i=1}^{\mu} \frac{1}{z_{i}} \cos ^{2} \angle\left(\xi, \eta_{i}\right) .
$$

The last equality follows from straightforward computation.

The directional steady state error variance with an arbitrary $F$ is

$$
V_{\xi}=\left\|\xi^{\prime}(I-F G) U\right\|_{2}^{2}
$$

and the optimal directional steady state error variance is

$$
\begin{aligned}
V_{\xi}^{*} & =\inf _{F, G-F G \in \mathcal{R} \mathcal{H}_{\infty}}\left\|\xi^{\prime}(I-F G) U\right\|_{2}^{2} \\
& =\inf _{Q \in \mathcal{R} \mathcal{H}_{\infty}, Q(0) \tilde{N}_{0}(0)=I}\left\|\xi^{\prime}\left(I-Q \tilde{N}_{0} \tilde{N}_{\nu} \cdots \tilde{N}_{1}\right) U\right\|_{2}^{2} .
\end{aligned}
$$


By following an almost identical derivation as the non-directional case, we can show that the same sequence $\left\{Q_{k}\right\}$ giving near optimal solutions there also gives near optimal solutions here for every $\xi \in \mathbb{R}^{m}$. Hence,

$$
V_{\xi}^{*}=V^{*}(\xi)=2 \sum_{i=1}^{\nu} \frac{1}{z_{i}} \cos ^{2} \angle\left(\xi, \eta_{i}\right) .
$$

We have thus established the following theorem.

THEOREM 2. Let $G$ 's nonminimum phase zeros be $z_{1}, z_{2}, \ldots, z_{\nu}$ with $\eta_{1}, \eta_{2}, \ldots, \eta_{\nu}$ being the corresponding Blaschke vectors of type II, then

$$
V^{*}=2 \sum_{i=1}^{\nu} \frac{1}{z_{i}}
$$

and

$$
V_{\xi}^{*}=V^{*}(\xi)=2 \sum_{i=1}^{\nu} \frac{1}{z_{i}} \cos ^{2} \angle\left(\xi, \eta_{i}\right) .
$$

This theorem says that to estimate a Brownian motion from its version distorted by an LTI system, the best achievable steady state error variance is proportional to the reciprocal sum of the nonminimum phase zeros of the LTI system. The best achievable directional steady state variance depends, in addition, on the directional characteristics of the nonminimum phase zeros.

5. Estimation under Brownian Noise. Consider the estimation problem considered in Sect. 3, but let us assume that the noise $n$ is a Brownian motion process instead of a white noise. In this case, the steady state variance of the estimation error is given by

$$
V=\|F N\|_{2}^{2}
$$

where $N(s)=\frac{1}{s} I$. Following similar arguments as in Sect. 3 , we get

$$
\begin{aligned}
V^{*} & =\inf _{F, G-F G \in \mathcal{R} \mathcal{H}_{\infty}, F(0)=0}\|F N\|_{2}^{2} \\
& =\inf _{Q \in \mathcal{R} \mathcal{H}_{\infty}, Q(0) \tilde{M}(0)=I}\|(I-Q \tilde{M}) N\|_{2}^{2} .
\end{aligned}
$$

Here we assume that $G=\tilde{M}^{-1} \tilde{N}$ is a left coprime factorization of $G$. Now assme that $G$ has antistable poles $p_{1}, p_{2}, \ldots, p_{\mu}$ with $\zeta_{1}, \zeta_{2}, \ldots, \zeta_{\mu}$ be the corresponding pole Blaschke vectors of type II. Then $\tilde{M}$ has factorization

$$
\tilde{M}=\tilde{M}_{0} \tilde{M}_{\mu} \cdots \tilde{M}_{1}
$$

where

$$
\tilde{M}_{i}(s)=I-\frac{2 \operatorname{Re} p_{i}}{p_{i}} \frac{s}{s+p_{i}^{*}} \zeta_{i} \zeta_{i}^{*}
$$


Now the problem becomes similar to the one considered in Sect. 4 with $\tilde{N}_{i}$ replaced by $\tilde{M}_{i}$. The following theorem is then obtained by using similar arguments as in Sect. 4 .

Theorem 3. Let $G$ 's antistable poles be $p_{1}, p_{2}, \ldots, p_{\mu}$ with $\zeta_{1}, \zeta_{2}, \cdots, \zeta_{\mu}$ being the corresponding pole Blaschke vectors of type II. Then

$$
V^{*}=2 \sum_{i=1}^{\mu} \frac{1}{p_{i}}
$$

and

$$
V_{\xi}^{*}=V^{*}(\xi)=2 \sum_{i=1}^{\mu} \frac{1}{p_{i}} \cos ^{2} \angle\left(\xi, \zeta_{i}\right)
$$

This theorem says that to estimate a signal from its version corrupted by a Brownian motion noise, the best achievable steady state error variance is proportional to the reciprocal sum of the antistable modes of the signal to be estimated. The best achievable directional steady state error variance depends, in addition, on certain directional characteristics of the antistable modes.

6. Estimate of White Noise. Consider the estimation problem as in Sect. 4, but now we assume that the signal to be estimated $u$ is a white noise, instead of a Brownian motion. In this case, the variance of the estimation error is given by

$$
V=\|I-F G\|_{2}^{2}
$$

If we want $V$ to be finite, we need to have $I-F(\infty) G(\infty)=0$, in addition to $F, I-F G \in \mathcal{R H}_{\infty}$. This requires $G(\infty)$, the direct feedthrough term of $G$, to be left invertible, which will be assumed. Equivalently, we need to have $F, F G \in \mathcal{H}_{\infty}$ and $F(\infty) G(\infty)=I$. Therefore

$$
\begin{aligned}
V^{*} & =\inf _{F, F G \in \mathcal{R} \mathcal{H}_{\infty}, F(\infty) G(\infty)=I}\|I-F G\|_{2}^{2} \\
& =\inf _{Q \in \mathcal{R} \mathcal{H}_{\infty}, Q(\infty) \tilde{N}(\infty)=I}\|I-Q \tilde{N}\|_{2}^{2} .
\end{aligned}
$$

Here we assume that $G=\tilde{M}^{-1} \tilde{N}$ is a left coprime factorization of $G$. Now let $G$ have nonminimum phase zeros $z_{1}, z_{2}, \ldots, z_{\nu}$ with $\eta_{1}, \eta_{2}, \ldots, \eta_{\nu}$ being the corresponding input Blaschke vectors of type I. Then $\tilde{N}$ has factorizations

$$
\tilde{N}=\tilde{N}_{0} \tilde{N}_{\nu} \cdots \tilde{N}_{1}
$$

where

$$
\tilde{N}_{i}=I-\frac{2 \operatorname{Re} z_{i}}{s+z_{i}^{*}} \eta_{i} \eta_{i}^{*}
$$


Now the problem becomes similar to the one considered in Sect. 3 with $\tilde{M}_{i}$ replaced

by $\tilde{N}_{i}$. The following theorem is then obtained by using the similar arguments as in Sect. 3 .

THEOREM 4. Let $G$ 's nonminimum phase zeros be $z_{1}, z_{2}, \ldots, z_{\nu}$ with $\eta_{1}, \eta_{2}, \ldots, \eta_{\nu}$ being the corresponding Blaschke vectors of type I, then

$$
V^{*}=2 \sum_{i=1}^{\nu} z_{i}
$$

and

$$
V_{\xi}^{*}=V^{*}(\xi)=2 \sum_{i=1}^{\nu} z_{i} \cos ^{2} \angle\left(\xi, \eta_{i}\right) .
$$

This theorem says that to estimate a white noise from its version distorted by an LTI system, the best achievable steady state error variance is proportional to the sum of the nonminimum phase zeros of the LTI system. The best achievable directional steady state variance depends, in addition, on the directional characteristics of the nonminimum phase zeros.

7. Concluding Remarks. This paper relates the performance limitations in four typical estimation problems to simple characteristics of the plants involved. By estimation problems we mean actually filtering problems here. The general estimation problems can include prediction and smoothing problems. We are now trying to extend the results in this paper to smoothing and prediction problems.

We have considered two type of noises and signals: while noise and Brownian motion. We are trying to extend our results to possibly other types of noises and signals.

\section{REFERENCES}

[1] B. D. O. Anderson and J. B. Moore, Optimal Filtering, Prentice-Hall, 1979.

[2] K. J. А́ström, Introduction to Stochastic Control Theory, Academic Press, 1970.

[3] J. Chen, L. QIU, And O. Toker, Limitation on maximal tracking accuracy, IEEE Trans. on Automat. Contr., 45(2000), pp. 326-331.

[4] G. C. Goodwin, D. Q. Mayne, And J. Shim, Trade-offs in linear filter design, Automatica, 31(1995), pp. 1367-1376.

[5] G. C. Goodwin, M.M. Seron, Fundamental design tradeoffs in filtering, prediction, and smoothing, IEEE Trans. Automat. Contr., 42(1997), pp. 1240-1251.

[6] H. KwakernaAk and R. Sivan, Linear Optimal Control Systems, Wiley-Interscience, New York, 1972.

[7] L. QIU AND J. Chen, Time domain characterizations of performance limitations of feedback control, Learning, Control, and Hybrid Systems, Y. Yamamoto and S. Hara, editors, Springer-Verlag, pp. 397-415, 1998.

[8] L. Qiu And E. J. Davison, Performance limitations of non-minimum phase systems in the servomechanism problem, Automatica, 29(1993), pp. 337-349. 
[9] Z. Ren, L. Qiu, And J. Chen, Performance limitations in estimation, Proc. 38th IEEE Conf. on Decision and Control, pp. 3204-3209, 1999.

[10] W. Rudin, Real and Complex Analysis, 3rd ed., McGraw-Hill, 1987.

[11] M. M. Seron, J. H. Braslavsky, and G. C. Goodwin, Fundamental Limitations in Filtering and Control, Springer, 1997.

[12] M. M. Seron, J. H. Braslavsky, D. G. Mayne, and P. V. Kokotovic, Limiting performance of optimal linear filters, Automatica, 35(1999), pp. 189-199. 